\title{
Derrame pleural: relato de um caso com difícil confirmação de diagnóstico etiológico
}

\section{Pleural effusion: report of a case with tough etiological diagnosis confirmation}

\author{
Thiago Gadelha Valle Oliveira ${ }^{1}$ \\ Rafael Rocha Rezende ${ }^{1}$ \\ Vinicius Costa Vieira ${ }^{2}$ \\ Jader Cunha de Azevedo ${ }^{3}$
}

Artigo

Original

Original

Paper

Recebido em $03 / 2012$

Relata-se o caso de uma paciente de 56 anos com histórico de derrame pleural (DP) à esquerda encaminhada para o ambulatório de cardiologia para investigação de insuficiência cardíaca (IC) como possível fator causal. Esse relato tem como objetivo trazer para discussão um caso comum na rotina médica, abordando a necessidade de conhecimento dos fatores etiológicos e diagnósticos da Síndrome de Derrame Pleural. Os critérios de Light funcionam como importante método de análise para diferenciação entre DP transudativo e exsudativo, sendo ainda fundamental a correlação com demais comorbidades encontradas na história clínica do paciente. Apesar de encontrados fatores de risco para IC, não se confirmou essa nosologia, sendo adequada uma melhor investigação diagnóstica.

Aprovado em $04 / 2013$

\section{Abstract}

This is a case report of a 56-year-old woman with a history of left sided pleural effusion referred to the cardiology clinic for investigation of heart failure. This report aims to bring to discussion a common case in routine care, addressing the need for knowledge of the etiological factors and diagnosis of pleural effusion syndrome. The Light's criteria are an important analysis method for the differentiation between transudative and exudative pleural effusion, and it is also fundamental to correlate them with other comorbidities found in the patient's clinical history. Although risk factors for heart failure were found, this nosology could not be confirmed, requiring a better diagnostic investigation.
Keywords:

Pleural effusion

Differential Diagnosis

Heart failure

\footnotetext{
${ }^{1}$ Acadêmico do $10^{\circ}$ período de Medicina - Centro Universitário de Volta Redonda - UniFOA - Volta Redonda - RJ

${ }^{2}$ Acadêmico do $9^{\circ}$ período de Medicina - Universidade Estácio de Sá - UNESA - Rio de Janeiro - RJ

${ }^{3}$ Médico Cardiologista, Mestre em Ciências Cardiovasculares, Professor Adjunto da Disciplina de Clínica

Médica - curso de Medicina - Centro Universitário de Volta Redonda - UniFOA - Rio de Janeiro - RJ.
} 


\section{Introdução}

O derrame pleural (DP) ocorre quando uma quantidade excessiva de líquido se acumula no espaço pleural, podendo ser decorrente de diversas causas. Apesar de a insuficiência cardíaca (IC) ser citada como a mais prevalente etiologia, muitas vezes outros fatores causais não são avaliados, comprometendo tanto o diagnóstico etiológico como o tratamento subseqüente. Portanto, torna-se fundamental o reconhecimento desta entidade nosológica, assim como esgotarmos a investigação diagnóstica para seu adequado manuseio.

\section{Metodologia}

O presente estudo consiste um relato de caso, cujas informações foram obtidas por meio de revisão de prontuário e dos resultados de exames laboratoriais da enferma, entrevista com a paciente e revisão da literatura.

O relato do caso foi enviado e aprovado pelo Comitê de Ética do Centro Universitário de Volta Redonda-RJ UniFOA.

\section{Relato do caso}

A seguir, relatamos o caso de uma paciente de 56 anos, sexo feminino, encaminhada ao ambulatório de cardiologia para investigação de IC como etiologia de um DP ocorrido há aproximadamente 2 meses da consulta. Histórico de 3 internações hospitalares nos seis meses antecedentes por estar apresentando febre, tosse, dispnéia em repouso e dor lombar. Na $3^{\mathrm{a}}$ internação, o diagnóstico de DP à esquerda foi feito através da radiografia de tórax e a paciente foi submetida à drenagem torácica, com posterior alívio dos sintomas. Apresentava, previamente, hipertensão arterial sistêmica, diabetes mellitus, artrite reumatóide e hipotireoidismo, além de história de depressão e hiperuricemia. Estava em uso de: losartana 50mg, hidralazina 50mg, levotiroxina sódica $50 \mathrm{mg}$, glimepirida $4 \mathrm{mg}$, metformina $850 \mathrm{mg}$, fluoxetina $20 \mathrm{mg}$, metotrexate $2,5 \mathrm{mg}$, ácido fólico $5 \mathrm{mg}$, prednisona $5 \mathrm{mg}$ e ácido acetilsalicílico 100mg. Fumou por 34 anos de 1 a 2 maços/dia tendo parado há 5 anos e negava uso de bebidas alcoólicas. Ao exame físico, apresentava bom estado geral, freqüência cardíaca de 60 batimentos por minuto, freqüência respiratória 16 incursões respiratórias por minuto, pressão arterial $114 \times 72 \mathrm{mmHg}$, temperatura axilar $36,7^{\circ} \mathrm{C}$, IMC $34,9 \mathrm{~kg} / \mathrm{m}^{2}$. $\mathrm{O}$ aparelho cardiovascular apresentava bulhas normofonéticas, ritmo cardíaco regular em 2 tempos sem sopros ou extrassístoles. $\mathrm{O}$ aparelho respiratório apresentava murmúrio vesicular universalmente audível sem ruídos adventícios, frêmito toracovocal presente bilateralmente e expansibilidade torácica normal. Trazia exames realizados previamente ao quadro clínico, que foram: Cintilografia de perfusão miocárdica sob estresse farmacológico com dipiridamol 8 meses antes sem evidências de isquemia, fração de ejeção do ventrículo esquerdo pelo Gated SPECT de 76\%; Ecocardiograma 18 meses antes com função sistólica global e segmentar do ventrículo esquerdo preservadas.

Durante a internação foram drenados $1000 \mathrm{~mL}$ de líquido pleural, mas não há relato de estudo citológico, citométrico ou bioquímico do líquido drenado.

Para melhor investigação diagnóstica, foram solicitados exames para pesquisa de BAAR, ácido úrico, creatinina, glicose em jejum, sódio, potássio, uréia, hemograma completo, T4 livre, TSH, EAS, hemoglobina glicosilada, teste tuberculínico, ultrassonografia dos rins e vias urinárias. Todos sem alterações significativas.

\section{Discussão}

O achado de DP constitui um diagnóstico sindrômico, requerendo, portanto, complementação diagnóstica para identificação de sua etiologia. Atualmente, o primeiro passo dessa investigação é realizado através da toracocentese diagnóstica e da determinação dos níveis pleurais de desidrogenase láctica (LDH) e proteínas.

A dosagem dessas substâncias foi proposta em 1972, quando Light et al ${ }^{1}$ criaram critérios para diferenciação do DP exsudativo do transudativo através da dosagem da LDH e proteínas totais do soro e do líquido pleural. Dessa forma, um DP exsudativo é assim classificado se preencher ao menos um dos seguintes crité- 
rios: relação entre proteínas do líquido pleural/ soro > 0,5; relação entre LDH do líquido pleu$\mathrm{ral} /$ soro $>0,6 ; \mathrm{LDH}$ do líquido pleural > 2/3 do valor de referência no soro. Caso não haja nenhum dos critérios mencionados, o derrame é classificado como transudativo.

O DP exsudativo ocorre quando há alteração nos fatores locais que regulam a formação e absorção do líquido pleural. Portanto, relacionado ao aumento da permeabilidade da microvascularização pleural e/ou obstrução linfática ou do mediastino ${ }^{2}$. Dentre suas principais causas, podemos citar infecções, neoplasias malignas e tromboembolia pulmonar ${ }^{3}$.

O transudato forma-se quando há alteração dos fatores sistêmicos que influenciam na produção e absorção do líquido pleural e as superfícies pleurais estão preservadas. Suas principais causas são insuficiência cardíaca, cirrose hepática e tromboembolia pulmonar ${ }^{3}$.

A maior parte dos transudatos é diagnosticada mais simplesmente, através de anamnese e exame físico compatíveis com doença sistêmica associados à avaliação radiológica e laboratorial do líquido pleural ${ }^{4}$. Entretanto, para derrames exsudativos, é necessária maior complementação diagnóstica, como descrição das características físicas, concentração de glicose, contagem diferencial de células, exames microbiológicos e citologia.

Dentre os exsudatos, a tuberculose pleural deve receber especial importância no nosso contexto, pois estudos mostraram que ela é responsável por metade dos derrames na cidade do Rio de Janeiro ${ }^{5-6}$, índice que pode ser semelhante para as cidades próximas, como Volta Redonda. Um dos novos marcadores de tuberculose que vem ganhando destaque devido a sua alta sensibilidade é a da adenosina deaminase (ADA) ${ }^{7}$. Entretanto, apesar de toda a investigação diagnóstica, sabe-se que cerca de 10 a $20 \%$ dos DPs podem permanecer sem diagnóstico etiológico definido após os exames convencionais, sendo conhecidos como derrames pleurais de origem indeterminada ${ }^{8 .}$

A IC é uma síndrome caracterizada pela presença, principalmente, de edema de membros inferiores, dispnéia paroxística noturna e ortopnéia, além de outros sinais e sintomas, como fadiga e noctúria. Ao exame físico, pode-se encontrar turgência de jugular, ritmo de galopes com presença de terceira bulha e estertores basais à ausculta pulmonar ${ }^{4}$. No caso relatado, a paciente apresentava-se sem qualquer desses sinais ou sintomas ao exame físico, além de apresentar exames prévios indicando função miocárdica normal, o que nos pode afastar a IC como primeira hipótese diagnóstica para etiologia do DP.

A paciente apresentava ainda outras comorbidades que são descritas como causadoras de DP. Este é reconhecido desde 1955 como possível manifestação sistêmica de artrite reumatóide, sendo esta manifestação mais comum em homens, com idade média e com sintomas articulares recentes ou em curso. O líquido pleural apresenta características não específicas de um exsudato, sendo altamente protéico e com a característica de apresentar glicose baixa, apesar de isto não ser invariável ${ }^{9}$.

O DP causado pelo hipotireoidismo é relatado como sendo uma etiologia rara. Normalmente, o derrame é pequeno, não inflamatório e possui características entre transudatos e exsudatos ${ }^{10}$.

Apesar de a paciente apresentar fatores de risco para IC, como hipertensão arterial, diabetes mellitus e história de tabagismo, exames diagnósticos não confirmaram esta nosologia. Logo, ressalta-se a necessidade da investigação apropriada frente a um quadro de derrame pleural, visto que na maioria das vezes a análise do líquido pleural vem a ser fundamental para o diagnóstico.

\section{Conclusão}

A Síndrome de Derrame Pleural nem sempre se constitui de um valor etiológico fácil de ser elucidado. É fundamental o médico estar atento quanto aos diversos fatores causais e ser capaz de estabelecer os critérios diagnósticos a serem realizados. Aliado a isso, a clínica do paciente e suas comorbidades devem ser somadas e levadas em conta na hora de estabelecer um diagnóstico correto. 


\section{Referências}

LIGHT R.W., MACGREGOR M.I., LUCHSINGER P.C., et al. Pleural effusion: The diagnostic separation of transudates and exudates. Ann Intern Med. 1972;77:507-13.

MARANHÃO B., SILVA JR C.T., CARDOSO G.P. Critérios bioquímicos para classificar transudatos e exsudatos pleurais. Pulmão RJ 2005;14(4):315-320.

LIGHT R.W. Pleural Effusion. N Engl J Med 2002;346(25):1971-7.

GENOFRE E., CHIBANTE A.M.S., MACEDO A.G. Derrame Pleural de Origem Indeterminada. J Bras Pneumol. 2006;32(Supl 4):S204-S210.

NEVES D.D. O valor da adenosina desaminase no diagnóstico diferencial dos derrames pleurais [Tese de Mestrado]. Rio de Janeiro: Universidade Federal do Rio de Janeiro; 1992.
SILVA JR C.T. Adenosina desaminase "versus" histopatológico pleural: avaliação da importância da toracocentese isolada para o diagnóstico da tuberculose pleural [Tese de doutorado]. Niterói (RJ): Universidade Federal Fluminense; 2000.

NEVES D.D., DIAS R.M., CUNHA A.J.L.A., et al. Rendimentos de variáveis clínicas, radiológicas e laboratoriais para o diagnóstico da tuberculose pleural. J Bras Pneumol 2004; 30(4) 409-16.

VILLENA V., LÓPEZ E.A., ECHAVE S.J., et al. Estudio prospectivo de 1.000 consecutivos pacientes con derrame pleural. Etiología del derrame y características de los pacientes. Arch Bronconeumol. 2002; 38:21-6.

CARMICHAEL D.S., GOLDING D.N. Rheumatoid pleural effusion with "R.A. cells" in the pleural fluid. Brit. med.J7.1967;2;814.

GOTTEHRER A., ROA J., STANFORD G.G., et al. Hypothyroidism and pleural effusions. Chest 1990;98;1130-1132. 